Résumés des conférences et travaux

\title{
Philologie et dialectologie grecques
}

\section{Laurent Dubois}

\section{OpenEdition \\ Journals}

\section{Édition électronique}

URL : https://journals.openedition.org/ashp/1142

DOI : $10.4000 /$ ashp. 1142

ISSN : 1969-6310

\section{Éditeur}

Publications de l'École Pratique des Hautes Études

\section{Édition imprimée}

Date de publication : 1 octobre 2011

Pagination : 71-72

ISSN : 0766-0677

\section{Référence électronique}

Laurent Dubois, "Philologie et dialectologie grecques », Annuaire de l'École pratique des hautes études (EPHE), Section des sciences historiques et philologiques [En ligne], 142 | 2011, mis en ligne le 21 juillet 2011, consulté le 06 juillet 2021. URL : http://journals.openedition.org/ashp/1142 ; DOI : https:// doi.org/10.4000/ashp. 1142 


\title{
PHILOLOGIE ET DIALECTOLOGIE GRECQUES
}

\author{
Directeur d'études : M. Laurent DuboIs
}

Programme de l'année 2009-2010 : I. Inscriptions grecques archaïques récemment publiées. II. Commentaire à l'Alexandra de Lycophron (suite).

Les conférences de cette année ont commencé par la présentation de documents récemment publiés. Nous avons d'abord étudié les defixiones d'Istros, dans le Pont gauche, publiées par A. Avram, C. Chiriac et I. Matei dans le BCH 2007 [2009], 383420. Il s'agit de documents intéressants pour leur onomastique et leur formulaire : on

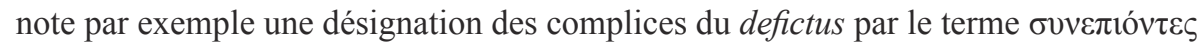
qui rappelle le participe ovvióv $\tau \varepsilon \varsigma$ de l'inscription d'Olbia IGDOP 101. Nous avons dû également faire un détour par la grande defixio de Pella en Macédoine, REG 1995, 190-197, qui présente un certain nombre de traits comparables.

Le second groupe de documents étudiés est constitué par les décrets thessaliens publiés par A. Tziafalias et Br. Helly dans le même fascicule du BCH 2007, p. 421482. Nous avons mis en évidence les traits dialectaux spécifiques et nouveaux : le

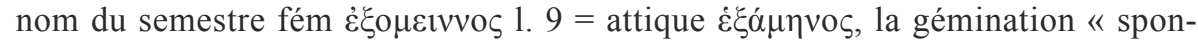

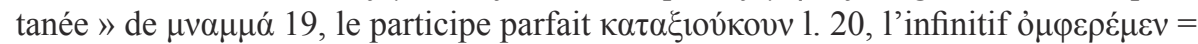

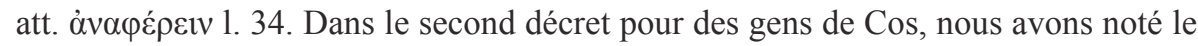

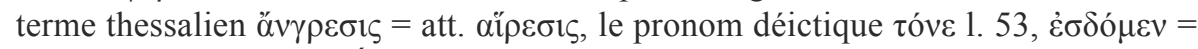

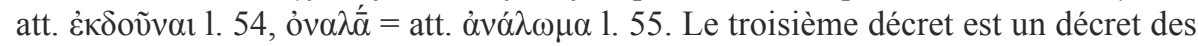
Molosses qui honore le peuple de Larissa : il est donc intéressant pour jauger l'état du dialecte épirote au second siècle avant notre ère. La forme d'infinitif $\tau$ นoṽ $\mu \varepsilon v 1.62$, si c'est bien la bonne lecture (on attendrait plutôt $\tau \nmid \mu \alpha \theta \tilde{\eta} \mu \varepsilon v$ comme dans d'autres textes épirotes), est un élément à prendre en compte pour la possibilité de l'existence d'un verbe $\tau$ น

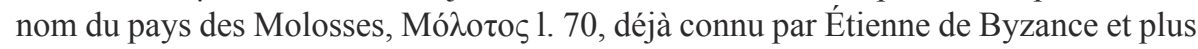

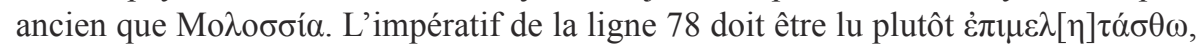

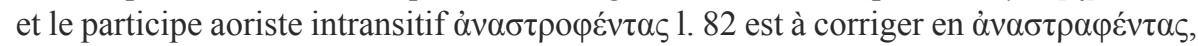
sauf à admettre une variante dialectale - $\rho$ o- de att. - $\rho \alpha-$.

Restant en Thessalie, nous avons rendu compte du livre d'A. Contogiannis,

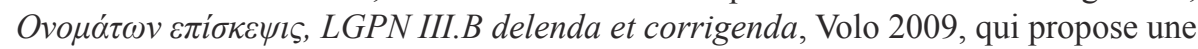
série remarquable de corrections ou de suppressions dans le recueil oxonien consacré à la Grèce centrale et en particulier à la Thessalie : l'auteur montre, après révision des pierres, qu'il faut ne citer les hapax et les noms rares de ce fascicule qu'avec circonspection et que certains d'entre eux sont imputables à des mélectures ou à des coupes de mots inadéquates.

Nous avons enfin étudié quelques épigrammes argiennes d'époque hellénistique intéressantes pour leur onomastique ou leur contenu historique. D'abord l'épigramme 
qui figure sur le tombeau du héros local Phoronée publiée par Olga Psychogyou, dans le Premier congrès sur la Grèce du Sud et de l'Ouest, Athènes 2006, p. 302. Le texte et ses allusions mythologiques s'inscrivent dans le cadre de la politique péloponnésienne du $\mathrm{II}^{\mathrm{e}}$ siècle $a$. C. Dans le même volume G. Kavvadias, p. 325-335, publie un certain nombre d'épitaphes d'époques variées retrouvées en remploi dans un cénotaphe d'époque romaine. Le texte le plus intéressant est l'épigramme funéraire, datable par l'écriture au tourant de notre ère, d'un boxeur nommé Dèmophilos qui a préféré mourir au combat que d'être un vaincu. Il est enterré aux frais de la cité d'Argos. Le distique final contient un remarquable dialogue entre Hadès et le Soleil : "Aı $\delta \alpha \varsigma$ 'A $\varepsilon \lambda i ́ \omega$.

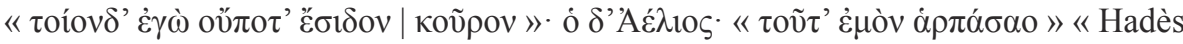
au Soleil "Je n'ai jamais vu tel jeune homme". Le Soleil : "Ce bien qui est mien tu me l'as dérobé" "».

Le fil conducteur de l'année a été une étude de l'ionien archaïque et en particulier des principaux textes, dont certains très récemment publiés, qui présentent des exemples de la lettre sampi T caractéristique de l'ionien d'Asie et très mal attesté dans l'épigraphie attique des plus hautes époques. Après avoir vainement chercher dans l'ouvrage d'I. Adiego, The Carian Language 2007, la possibilité d'une origine carienne pour ce signe, nous avons admis l'idée remontant à Claude Brixhe, $B S L$ 1082, selon laquelle cette lettre serait un tau diacrité créé pour noter une affriquée valant à peu près /ts/. Rappelons aussi qu'elle est attestée dans un document phrygien $\mathrm{du} \mathrm{VII} / \mathrm{VI}^{\mathrm{e}}$ s. $a$. C. : Brixhe-Lejeune, Corpus des inscriptions paléo-phrygiennes 1984, p. 250.

Nous avons entamé l'inventaire à partir de la dédicace à l'Athéna d'Assésos du

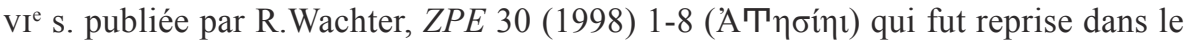
volume Milet VI, 3, 1278, dédicace qui fut récemment corroborée par une autre dédicace de la fin du VI ${ }^{\mathrm{e}}$ s. $a$. C. publiée par G. Petzl, ZPE 169 (2009), p. 89-92, fig. 1-5 :

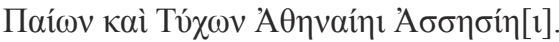

Nous avons ensuite étudié les deux textes importants d'Halicarnasse, Syll. ${ }^{3}, 45$ et 46, dans le premier desquels nous avons pu constater que le sampi apparaît d'une façon non systématique en alternance avec les deux sigma et uniquement dans des anthropo-

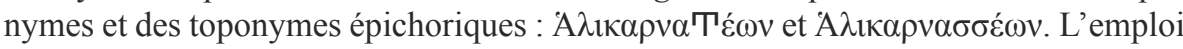
du sampi est donc plutôt un vestige fossilisé et résiduel, ce qui n'est pas le cas dans l'inscription d'Éphèse, I.Eph. 1 (= Schwyzer, $D G E$ 707), évoquant la fondation du temple par Crésus : dans ce texte le sampi est utilisé pour la notation d'un stade érodé

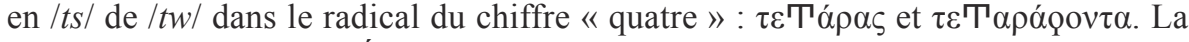
présence dans ce texte d'Éphèse d'une formule indiquant la soustraction à un chiffre

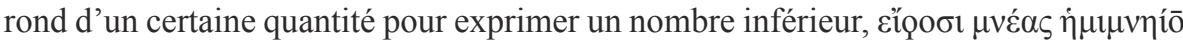
$\delta \varepsilon \circ \mu \varepsilon v^{\prime} \alpha \varsigma=19$ mines et demie, nous a conduits à examiner quelques exemples anciens de ce comput en particulier dans les comptes de l'Acropole $I G \mathrm{I}^{3}, 474-475$, où apparaît non plus le moyen mais l'actif $\delta \varepsilon ́ o v \sigma \alpha$. De là nous nous sommes intéressés à la

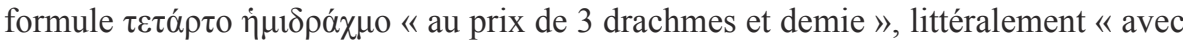
la quatrième drachme dont on ne compte que la moitié », qui se rencontre aussi dans un vieux texte ionien et plus exactement phocéen, celui de Pech Maho en Languedoc, qu'avaient publiées M. Lejeune et J. Pouilloux, dans la $R A N$ de 1988.

L'enquête se continuera l'année suivante. 